\title{
A diffusion-weighted imaging based diagnostic system for early detection of prostate cancer
}

\author{
Ahmad Firjani ${ }^{1,2}$, Ahmed Elnakib ${ }^{1}$, Fahmi Khalifa ${ }^{1}$, Georgy Gimel’farb ${ }^{3}$, Mohamed Abou El-Ghar ${ }^{4}$, \\ Adel Elmaghraby ${ }^{2}$, Ayman El-Baz ${ }^{1^{*}}$ \\ ${ }^{1}$ BioImaging Laboratory, Bioengineering Department, University of Louisville, Louisville, USA \\ ${ }^{2}$ Department of Computer Engineering and Computer Science, University of Louisville, Louisville, USA \\ ${ }^{3}$ Department of Computer Science, University of Auckland, Auckland, New Zealand \\ ${ }^{4}$ Radiology Department, Urology and Nephrology Center, University of Mansoura, Mansoura, Egypt \\ Email: ${ }^{\text {aselba01@exchange.louisville.edu }}$
}

Received 18 January 2013; revised 21 February 2013; accepted 1 March 2013

\begin{abstract}
A new framework for early diagnosis of prostate cancer using Diffusion-Weighted Imaging (DWI) is proposed. The proposed diagnostic approach consists of the following four steps to detect locations that are suspicious for prostate cancer: 1) In the first step, we isolate the prostate from the surrounding anatomical structures based on a Maximum A Posteriori (MAP) estimate of a new log-likelihood function that accounts for the shape priori, the spatial interaction, and the current appearance of prostate tissues and its background (surrounding anatomical structures); 2) In order to take into account any local deformation between the segmented prostates at different $b$-values that could occur during the scanning process due to local motion, a non-rigid registration algorithm is employed; 3) A KNN-based classifier is used to classify the prostate into benign or malignant based on three appearance features extracted from registered images; and 4) The tumor boundaries are determined using a level set deformable model controlled by the diffusion information and the spatial interactions between the prostate voxels. Preliminary experiments on 28 patients (17 malignant and 11 benign) resulted in $100 \%$ correct classification, showing that the proposed method is a promising supplement to current technologies (biopsy-based diagnostic systems) for the early diagnosis of prostate cancer.
\end{abstract}

Keywords: Prostate Cancer; 3D Markov-Gibbs Random Field; Nonrigid Registration; Diffusion-Weighted Imaging

\section{INTRODUCTION}

Prostate cancer is a major health problem, and the most

\footnotetext{
${ }^{\text {ga* }}$ Corresponding author.
}

frequently diagnosed malignancy in the American male population [1]. Recent prostate cancer studies reported an estimated 241,740 new cases and a mortality rate of close to 28,170 in 2012 [2]. Fortunately, early diagnosis of prostate cancer increases the survival rate of the patients [3].

\subsection{Current Imaging Modalities for Prostate Cancer Diagnosis}

Currently, there are different techniques that are used for early diagnosis of prostate cancer. However, the accuracy of these techniques are clearly unsatisfactory. For example, Prostate Specific Antigen (PSA) screening doesn't offer accurate information about the location and extent of the lesion(s) [4]. In addition, PSA is associated with a high risk of over diagnosis of prostate cancer.

On the other hand, imaging tests using different imaging modalities, such as Transrectal Ultrasound (TRUS) [5], Computed Tomography (CT) [6], MR Spectroscopy (MRS) [7], Dynamic-Contrast Enhanced Magnetic Resonance Imaging (DCE-MRI) [8], and Diffusion-Weighted Imaging (DWI) [9] are still critically needed. TRUS imaging [10] is widely used for guided needle biopsy due to the real time nature of the imaging system, ease of use, and portability. However, TRUS images have low Signal-to-Noise Ratio (SNR) making it difficult to detect malignant tissues [11]. Another traditional imaging modality is CT. It is widely used for diagnosis and followup of prostate cancer [12]. However, it has poor softtissue contrast resolution that does not allow precise distinction of the internal or external anatomy of the prostate and thus CT images have shown limited specificity for prostate diagnosis [13].

On the other hand, MR image-based modalities, such as T2-weighted MR, MRS, DCE-MRI, and DWI, have also been widely employed for early detection of prostate cancer [14]. Despite widely use of T2-weighted MR im- 
aging for prostate cancer, the technique is limited by unsatisfactory sensitivity and specificity for cancer detection and localization [15]. To improve the diagnostic performance of MR imaging in evaluations for prostate cancer, various other techniques have been applied. MRS provides metabolic information about prostate tissue by demonstrating the relative concentration of chemical compounds. However, MRS has its own limitations, such as the need of an additional software and longer acquisition time [16], which lead to increased costs and decreased throughput. Furthermore, MRS suffers from lack of spatial resolution. In addition, signal from periprostatic fat and seminal vesicles can distort spectral waveforms [14]. DCE-MRI has been recently suggested for improved visualization and localization of the prostate cancer [17]. It provides valuable pathologic and anatomical information. However, DCE-MRI has the drawback of intravenous contrast agent (e.g., gadolinium) administration which is harmful to the kidney [18] and requires a longer setup time.

Recently, DWI has emerged as an imaging modality that has shown more capabilities in determining the size and the shape of the prostate gland and localizing the cancer foci [19]. DWI is non-contrast functional imaging technique, whereby the image contrast is determined by the random microscopic motion of water protons, i.e., the Brownian motion [19]. Moreover, DWI has the distinct advantage of being acquired very rapidly, without the use of any intravenous contrast material or specialized hardware, and this is the main motivation behind this work.

\subsection{Clinical Studies for Prostate Cancer Diagnosis Using DWI}

In recent years, a growing number of clinical studies [1930] have evaluated the utility of DWI, either in combination with or in comparison with other MRI techniques, for the detection of prostate cancer. These studies have reported various sensitivities and specificities of cancer diagnosis.

Earlier studies $[19,20]$ have investigated the abilities of DWI for prostate cancer diagnosis using an endorectal coil. However, the reported results demonstrated low diagnostic sensitivity. To increase the sensitivity of diagnosis, Shimofusa et al. [21] suggested the addition of strong magnetic field gradient pulses ( $b$-values) to the pulse sequence instead of using endorectal coil. In their study [21], they detected the tumor in the central zone of the prostate in five of eight total patients using DWI with strong magnetic field gradient pulses. Alternatively, the compared diagnostic results with T2-weighted imaging, detected the tumor only in one of the eight patients. Since then, DWI was used for the detection of cancerous tissue in later studies [22-30]. For example, Tan et al. [30] compared the performance of T2-weighted MRI, DCEMRI, and DWI for the detection of cancer within the prostate gland. In their study they reported that DWI alone showed better specificity than DCE-MRI alone. It is also showed better overall specificity than combined DWI and T2-weighted imaging.

To the best of our knowledge, there are a very limited number of image-based approaches for automated computer-aided diagnosis of prostate cancer using DWI. These related works are discussed in the following section.

\subsection{Image-Based Computer-Aided Diagnostic (CAD) Systems for Prostate Cancer Detection}

In literature, a limited number of CAD systems for prostate cancer diagnosis have been proposed. For example, Chan et al. [31] proposed an in-vivo CAD system using multimodal MRI to estimate malignancy likelihood in the peripheral zone. They constructed statistical maps from T2-weighted MRI, DWI, and Proton Density (PD) images. These statistic maps were combined with textural and anatomical features of prostate cancer areas in order to detect the cancerous regions. However, this study doesn't include benign regions. Huisman et al. [32] developed a CAD system for prostate lesion classification using a Hessian-based blob detection algorithm [33]. Results showed an accuracy of 92\% in classification within the peripheral region and an accuracy of $83 \%$ in classification within transitional zones of the prostate. However, their study focused on the peripheral and transitional zones of the prostate gland and excluded central zones in which up to $30 \%$ of prostate cancers can occur.

Viswanath et al. [34] generated similar likelihood maps by combining information from multimodal MR images using mathematical descriptors. Their study showed, on a voxel basis, that the discrimination between benign and malignant tissue is feasible with good performances. The unsupervised classification by kmeans clustering achieved an accuracy of $77 \%$. Unfortunately, the corresponding slice still needs to be selected between different modality. A study by Langer et al. [35] focused on the peripheral zone of the prostate gland and excluded the central and transitional zones. However, detailed anatomic studies have suggested that $70 \%$ of cancers arise in the peripheral zone of the prostate, but up to $30 \%$ of prostate cancers occur between transition zones and the central zone of the prostate [36].

To increase the sensitivity of diagnosis, accurate delineation of the prostate region is mandatory. Basically, manual outlining of the prostate borders is the most accurate segmentation that enables precise determination of the prostate volume. However, it is prohibitively time 
consuming and is prone to intra- and inter-observer variability. Traditional edge detection methods (e.g., [39]) are unable to extract the correct boundaries of the prostate since the gray-level distributions of the prostate and the surrounding organs are hardly distinguishable. Therefore, other automated segmentation methods are desirable. However, multiple challenges stemming from 1) the large variations of prostate shape within a specific time series as well as across subjects; 2) lack of strong edges and diffused prostate boundaries; and 3) the similar signal-intensity profile of the prostate and surrounding tissues, complicates the segmentation process.

The most successful known approaches (e.g., [37-45]) have addressed the segmentation challenges of the prostate by modeling object appearance and shape. In particular, Zhu et al. [40] used a combination of an Active Shape Model (ASM) and 3D statistical shape modeling to segment the prostate. Toth et al. [41] presented an algorithm for the automatic segmentation of the prostate in multi-modal MRI. Their algorithm starts by isolating the Region-Of-Interest (ROI) from MRS data. Then, an ASM within the ROI is used to obtain the final segmentation. Klein et al. [42] presented an atlas-based segmentation approach to extract the prostate from MR images. The segmentation of the prostate is obtained as the average of the best-matched registered atlas set to the test image (image to be segmented). Recently, Vikal et al. [43] used a priori knowledge of prostate shape to detect the contour in each slice and then refined them to form a 3D prostate surface. Martin et al. [44] developed an atlas-based approach for segmenting the prostate from 3D MR images by mapping probabilistic anatomical atlas to the test image. The resulting map is used to constrain a deformable model-based segmentation framework.

\subsection{Current Limitations and Motivation for Our Proposal}

The above-mentioned CAD systems for analyzing DWI are not sufficiently accurate and reliable for several reasons:

1) The majority of CAD systems used multimodal MRI which is cost inefficient [45].

2) The majority of these studies require user interaction to select a ROI (a small window) around the prostate. Unfortunately, such approaches not only prone to interobserver variability, but also ROI selection biases the final decision by over- or under-estimating the problem in the entire graft, just as with biopsy.

3) Automated prostate segmentation methods have one of the following limitations:

- Deformable model-based methods without adequate appearance and shape priors fail under excessive noise, poor resolution, diffused boundaries, or oc- cluded shapes in the images;

- Segmentation based only on the shape prior still results in large errors caused by discontinuities in object boundaries, large image noise, and other inhomogeneities;

- Parametric shape-based models are unsuitable for discontinuous prostate objects due to a very small number of distinct landmarks.

4) The majority of CADs assumes that the prostates (prostate contours) remain exactly the same from scan to scan. However, prostate contours may not always exactly match due to patient movement or breathing effects; therefore, image registration schemes should be applied first before ROI selection/segmentation.

To overcome these limitations, we propose an automatic framework for analyzing DWI images building on our previous work in [46-48]. The proposed approach consists of the following steps as shown in Figure 1:

1) Segmentation of the prostate from DWI (Section 2.1) based on a Maximum a Posteriori (MAP) estimate of a new likelihood function that accounts for both appearance features of the prostate (Section 2.1.1) and their 3D spatial voxel interactions (Section 2.1.2), as well as a 3D shape prior (Section 2.1.3).

2) A non-rigid registration approach is employed to account for any local deformation that could occur in the prostate during the scanning process based on the solution of the Laplace equation (Section 2.3).

3) KNN classifier to classify the prostate into benign or malignant based on three appearance features extracted from registered images (Section 3.2).

\section{MATERIALS AND METHODS}

In this paper we introduce a new, automated, and noninvasive framework for early diagnosis of prostate cancer from DWI. Figure 1 demonstrates the steps of the proposed CAD system. Below, we will illustrate each of these steps.

\subsection{Segmentation of the Prostate Using a Joint MGRF Model}

The segmentation of the prostate is a challenge, since the gray-level distribution of the prostate and surrounding organs is not highly distinguishable and because of the anatomical complexity of prostate. This stage proposes a powerful framework for prostate segmentation based on a learned shape model and an identifiable joint MarkovGibbs Random Field (MGRF) model of DWI and "object-background" region maps.

The joint-MGRF model is fundamentally a model that relates the joint probability of an image and its objectbackground region map, to geometric structure and to the energy of repeated patterns within the image. The basic 


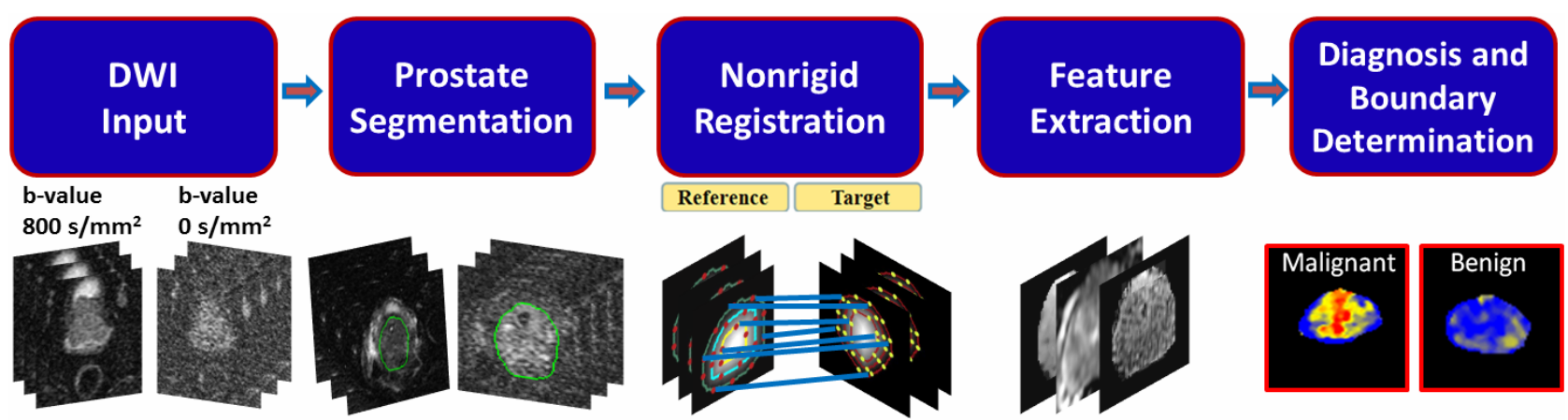

Figure 1. Flowchart of the proposed CAD system for automatic detection of cancer from 3D DWI.

theory behind such models is that they assume that the signals associated with each pixel depend on the signals of the neighboring pixels, and thus explicitly take into account their spatial interactions, and other features, such as the shape.

Let $\boldsymbol{Q}=\{0,1, \cdots, Q-1\}, \quad \boldsymbol{L}=\{o b, b g\}$, and $\boldsymbol{U}=[0,1]$ be a set of $\boldsymbol{Q}$ integer gray-level, a set of object ("ob") and background (" $b g$ ”) labels, and a unit interval, respectively. Let a 3D arithmetic grid

$$
\begin{aligned}
\boldsymbol{R}= & \{(x, y, z): x=0,1, \cdots, X-1 ; \\
& y=0,1, \cdots, Y-1 ; z=0,1, \cdots, Z-1\}
\end{aligned}
$$

support a grayscale DWI $\boldsymbol{g}: \boldsymbol{R} \rightarrow \boldsymbol{Q}$ and their binary region maps $\boldsymbol{m}: \boldsymbol{R} \rightarrow \boldsymbol{L}$, and probabilistic shape model $\boldsymbol{s}: \boldsymbol{R} \rightarrow \boldsymbol{U}$. The shape model allows for registering (aligning) 3D prostate DWI. The DWI data $g$ and their region maps $\boldsymbol{m}$ are described with a joint probability model [49,50]:

$$
P(\boldsymbol{g}, \boldsymbol{m})=P(\boldsymbol{g} \mid \boldsymbol{m}) P_{h}(\boldsymbol{m})
$$

where $P_{h}(\boldsymbol{m})$ is a $2^{\text {nd }}$-order MGRF of region maps and $P(\boldsymbol{g} \mid \boldsymbol{m})$ is a conditionally independent random field of image intensities given the map. The map model

$P_{h}(\boldsymbol{m})=P_{s}(\boldsymbol{m}) P(\boldsymbol{m})$ has two parts: a shape prior probability being a spatially variant independent random field of region labels $P_{s}(\boldsymbol{m})$, for a set of co-aligned training DWI data, and a $2^{\text {nd }}$-order MGRF model $P(\boldsymbol{m})$ of a spatially homogeneous evolving map.

The Bayesian MAP estimate of the map, given the DWI data $g, m^{*}=\arg \max L(g, \boldsymbol{m})$ maximize the loglikelihood function:

$$
L(\boldsymbol{g}, \boldsymbol{m})=\log (P(\boldsymbol{g} \mid \boldsymbol{m}))+\log \left(P_{h}(\boldsymbol{m})\right)
$$

In this work we focus on accurate identification of the spatial interaction between the prostate voxels $P(\boldsymbol{m})$, and the intensity distribution for the prostate tissues, $P(\boldsymbol{g} \mid \boldsymbol{m})$, and the prior distribution $P_{s}(\boldsymbol{m})$ of the prostate shape, as shown in Figure 2.

To perform the initial prostate segmentation, a given 3D DWI is aligned to one of the training 3D DWI. The

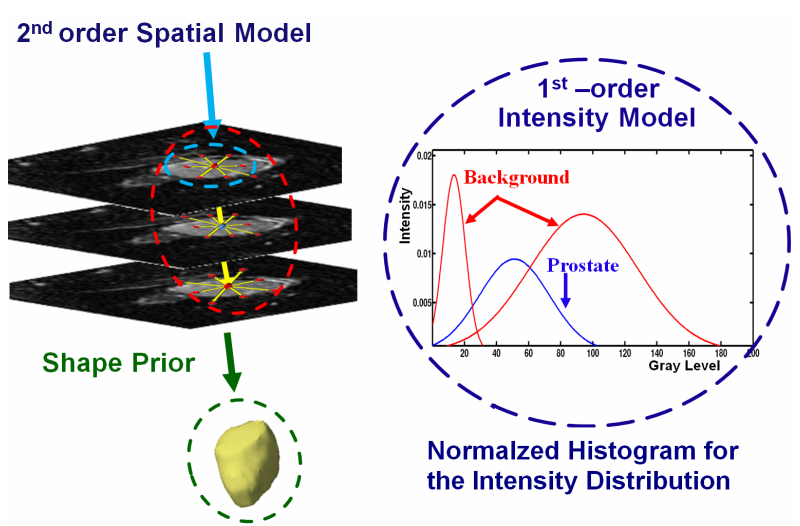

Figure 2. Aligning a 3-D joint Markov-Gibbs random field model with shape prior of DWI.

shape model provides the voxel-wise object and background probabilities being used, together with the conditional image intensity model $P(\boldsymbol{g} \mid \boldsymbol{m})$, to build an initial region map. The final Bayesian segmentation is performed using the identified joint MGRF model of the DWI and region maps.

\subsubsection{Conditional Intensity Model}

The specific visual appearance of the prostate in each data set to be segmented is taken into account by modeling a marginal gray level distribution with a Linear Combination of Discrete Gaussians (LCDG) [50,51]. Close approximation with LCDG separates each factor of the joint empirical gray level distribution,

$P(\boldsymbol{g})=\prod_{(x, y) \in R} p_{\text {mix }}\left(g_{x, y}\right)$, into two (object and background) components, $(p(q \mid \lambda) ; q \in \boldsymbol{Q}$, and $\lambda \in \boldsymbol{L})$. The LCDG modeling restores transitions between these components more accurately than conventional mixtures of only positive Gaussians, thus yielding a better initial region map formed by voxel-wise classification of the image gray values, the similar intensity profile of the prostate and surrounding tissues.

\subsubsection{Spatial Voxel Interaction Model}

In order to overcome noise effect and to ensure the homogeneity of the segmentation, spatially voxel interac- 
tions between the region labels are also taken into account using the popular Potts model, i.e., the MGRF with the nearest voxel 26-neighborhood (see Figure 3).

A generic MGRF of region maps accounts only for pairwise interaction between each region label and its characteristic neighbors. Generally, the interaction structure and the Gibbs potentials can be arbitrary and are identified from the training data.

By symmetry considerations, we assume that the potentials are independent of relative orientation of each voxel pair and depend only on intra- or inter-region position (i.e. whether the labels are equal or not). Under these restrictions, it is the $3 \mathrm{D}$ extension of the conventional auto-binomial, or Potts model differing only in that the potentials are estimated analytically.

The 26-neighborhood has three types of symmetric pairwise interactions specified by the absolute distance $a$ between two voxels in the same and adjacent MRI slices ( $a=1, \sqrt{2}$, and $\sqrt{3}$, respectively): 1) the closest pairs with the inter-voxel $\boldsymbol{N}_{1}=\{(1,0,0),(0,1,0),(0,0,1)\}$ coordinate offsets; 2$)$ the diagonal pairs with the offsets $\boldsymbol{N}_{\sqrt{2}}=\{(0,1, \pm 1),(1,0, \pm 1),(1, \pm 1,0)\}$; and 3) the farthest diagonal pairs with the offsets $\boldsymbol{N}_{\sqrt{3}}=\{(1, \pm 1, \pm 1)\}$. The Gibbs potentials of each type are bi-valued because only label coincidence is accounted for: $\boldsymbol{V}_{a}=\left\{V_{a, e q} ; V_{a, n e}\right\}$ where $V_{a, e q} \equiv V_{a}\left(l, l^{\prime}\right)$ if $l=l^{\prime}$ and $V_{a, n e} \equiv V_{a}\left(l, l^{\prime}\right)$ if $l \neq l^{\prime} ; \quad a \in \boldsymbol{A}=\{1, \sqrt{2}, \sqrt{3}\}$. Then the MGRF model of region maps is as follows [52,53]:

$$
P_{h}(\boldsymbol{m})=\frac{1}{z} \exp \sum_{(x, y, z) \in \boldsymbol{R}} \sum_{a \in \boldsymbol{A}} \sum_{(\xi, \eta, k) \in N_{a}} V_{a}\left(m_{x, y, z}, m_{x+\xi, y+\eta+z+k}\right)
$$

where $Z$ is the normalizing factor (partition function).

To identify the MGRF in Eq.1, approximate analytical maximum likelihood estimate of the 3D Gibbs potentials, $V_{a, e q}$ and $V_{a, n e}$ are derived in line with [52]:

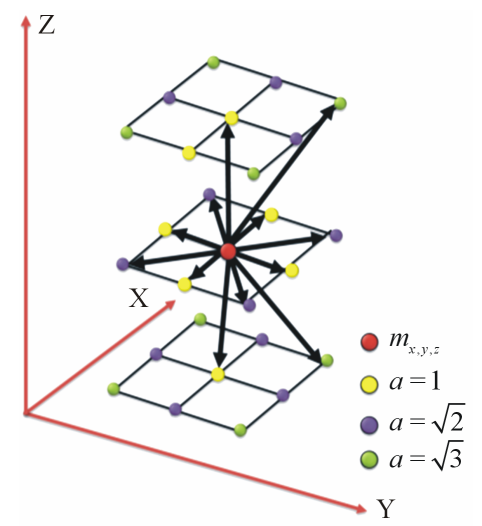

Figure 3. Pairwise voxel interaction for 26 neighborhood system in a 3D GGMRF. The reference voxel is shown in red.

$$
V_{a, e q}=-V_{a, n e}=2\left(f_{a, e q}(m)-\frac{1}{2}\right)
$$

where, $f_{a, e q}(m)$ denotes the relative frequency of the equal labels in the equivalent voxel pairs

$$
\begin{gathered}
\{((x, y, z),(x+\xi, y+\eta, z+\kappa)):(x, y, z) \in \boldsymbol{R} ; \\
\left.(x+\xi, y+\eta, z+\kappa) \in \boldsymbol{R} ;(\xi, \eta, \kappa) \in \boldsymbol{N}_{a}\right\}
\end{gathered}
$$

of a region map $m$ of a given DWI aligned in accord with the prior shape model.

\subsubsection{Probabilistic Shape Model}

To enhance the segmentation accuracy, the expected shape of the goal object is constrained with a soft probabilistic 3D prostate shape model. Initially, a training database collected from different subjects are co-aligned by rigid, affine 3-D transformations. The shape prior is a spatially variant independent random field of region labels:

$$
P_{s}(\boldsymbol{m})=\prod_{(x, y, z) \in \mathbf{R}} s_{m x, y, z}
$$

where is the empirical probability that the voxel $(x, y, z)$ belongs to the prostate ( $L=$ " ob") or the background ( $L=$ " $b g$ ") given the map. To enhance the segmentation of the current prostate volume, the prior probabilistic shape model is updated by adding the previous segmented 3D prostate data to the prior calculated shape model. The proposed prostate segmentation process can be summarize as follows:

- Perform an affine alignment of a given 3D MRI to an arbitrary prototype prostate from the training set using mutual information [54] as a similarity measure to obtain the learned probabilistic shape model $P_{s}(\boldsymbol{m})$;

- Estimate the conditional intensity model $P(\boldsymbol{g} \mid \boldsymbol{m})$ by identifying the bimodal LCDG;

- Use the intensity model found and the learned probabilistic shape model to perform an initial segmentation of the prostate, i.e., to form an initial region map;

- Use the initial region map to estimate the potential for the Potts model and to identify the MGRF model $P(\boldsymbol{m})$ of region maps;

- Improve the region map using voxel-wise stochastic relaxation (Iterative Conditional Mode (ICM) [55]) through successive iterations to maximize the log likelihood function of Eq.1 until the log likelihood remains almost the same for two successive iterations;

- Output: The 3D prostate segmentation is the final estimate $\boldsymbol{m}$.

\subsection{Performance Evaluation of the Proposed Segmentation Algorithm}

The proposed segmentation is evaluated based on char- 
acterizing the agreement (Figure 4(a)) and the Average Perpendicular Distance (APD) between the segmented and ground truth contours (Figure 4(b)). To evaluate the performance, we measured True Positive (TP), True Negative (TN), False Positive (FP), and False Negative (FN) segmentation (Figure 4(a)). Let $C$ and $G$ denote the segmented region and its "ground truth" counterpart, respectively.

Let $|z|$ denote the volume (in the number of voxels) of a region $z$. Then, $\mathrm{TP}=|C \cap G|$ is the overlap between $C$ and $G, \mathrm{FP}=|C-C \cap G|$ is the difference between $C$ and TP; and $\mathrm{FN}=|G-C \cap G|$ is the difference between $G$ and TP; and $\mathrm{TN}=|R-C \cup G|$.

The Positive Predictive Value (PPV), Sensitivity (Sens), Dice Similarity Coefficient (DSC), and the average segmentation error $\left(E_{\text {avg }}\right)$ are defined as:

$$
\begin{gathered}
\mathrm{PPV}=\frac{\mathrm{TP}}{\mathrm{TP}+\mathrm{FP}} \\
\text { Sens }=\frac{\mathrm{TP}}{\mathrm{TP}+\mathrm{FN}} \\
\mathrm{DSC}=\frac{2 \times \mathrm{TP}}{2 \times \mathrm{TP}+\mathrm{FP}+\mathrm{FN}} \\
E_{\text {avg }}=\frac{\mathrm{FP}+\mathrm{FN}}{\mathrm{FN}+\mathrm{TP}}
\end{gathered}
$$

\subsection{Nonrigid Registration}

Due to patient breathing and local movement, accurate registration is a main issue in DWI. In this paper, the nonrigid motion of the DWI data at different b-values is compensated for by using our developed registration approach that is based on the solution of the second-order partial differential Laplace equation [56]:

$$
\nabla^{2} \gamma=\frac{\partial^{2} \gamma}{\partial^{2} x^{2}}+\frac{\partial^{2} \gamma}{\partial^{2} y^{2}}=0
$$

for a scalar function $\gamma(x, y)$ between the target and the reference prostate objects. The solution of a planar Laplace equation between two boundaries results in intermediate equipotential surfaces (dashed lines in Figure 5) and streamlines that establish natural point-to-point correspondences and are everywhere orthogonal to all the equipotential surfaces (see e.g., the line connecting the points $B_{a i}$ and $B_{a i}$ in Figure 5). Based on solving the Laplace equation, we perform the non-rigid registration as follows:

1) Generate the distance maps inside the prostate regions as shown in Figures 6(a) and (b).

2) Use these distance maps to generate equispaced isocontours as shown in Figures 6(c) and (d).

3) Solve the Laplace equation between respective reference and target iso-contours to find the point-to-point correspondence.

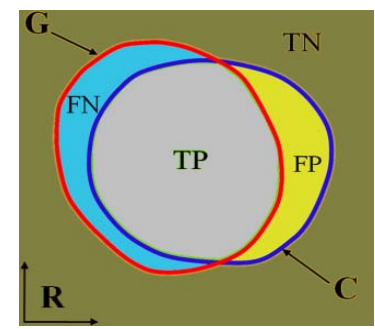

(a)

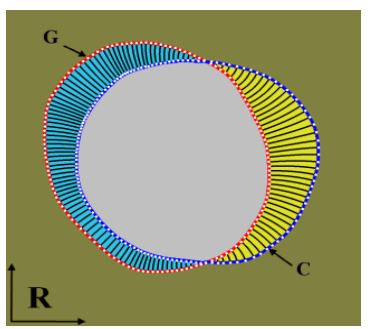

(b)
Figure 4. 2-D schematic illustration of measuring segmentation errors (a) and (b) perpendicular distances (black lines) (b) between the ground truth $\mathrm{G}$ and automatic segmentation $\mathrm{C}$.

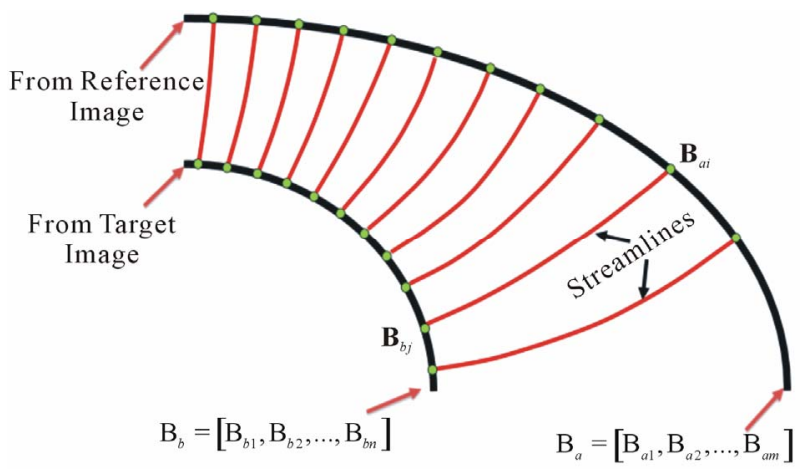

Figure 5. 2-D illustration of co-allocation of point-to-point correspondences between two borders by a potential field.

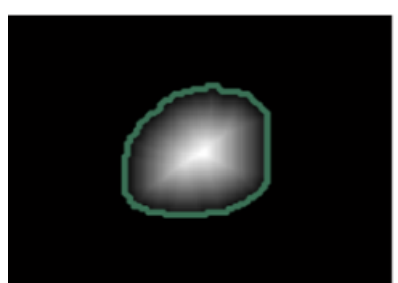

(a)

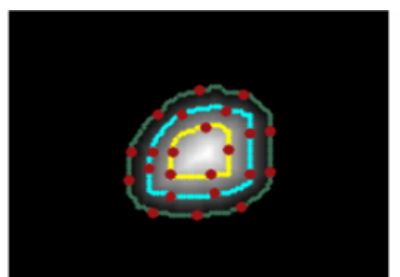

(c)

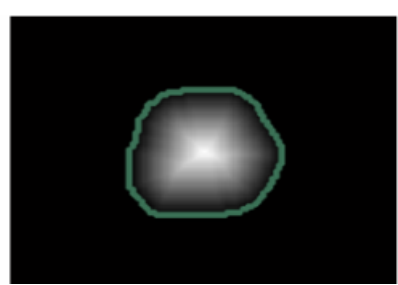

(b)

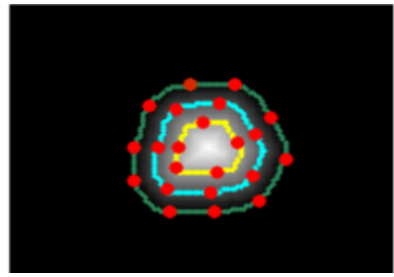

(d)
Figure 6. The distance maps (a), (b) and the iso-contours (c), (d) of the two prostates.

\subsection{Color Map Generation and Tumor Boundary Determination}

To characterize the physiological data, color-coded maps that illustrate the propagation of diffusion in the prostate tissues are constructed. To construct the initial color maps, we have to estimate the changes in image signals $\delta_{x, y, z}$ due to the Brownian motion. These changes are estimated from the constructed normalized diffusion as 
the difference between the signals of image sequences at two different $b$-values. DWI is performed with at least two $b$ values, including a $b$ value of $0 \mathrm{sec} / \mathrm{mm}^{2}$ and a higher $b$ value of $500-1000 \mathrm{~s} / \mathrm{mm}^{2}$ depending on the body region or organ being imaged [57]. At $b=0 \mathrm{~s} / \mathrm{mm}^{2}$, there is no diffusion sensitizing gradient with free water molecules have high signal intensity. We used $b=800$ $\mathrm{s} / \mathrm{mm}^{2}$ because imaging of solid organs requires high $b$ value specially in prostate and using high $b$ values allows differentiation of areas of restricted from the normal high signal at the peripheral zone. During our trials we found the $b=800 \mathrm{~s} / \mathrm{mm}^{2}$ allows lesions differentiation with least degradation of image quality as the image quality decrease with the high $b$ values.

To preserve continuity (remove inconsistencies), the initial estimated $\delta_{x, y, z}$ values are considered as samples from a Generalized Gauss-Markov Random Field (GGMRF) image model [58] of measurements with the 26-voxel neighborhood (Figure 3). Continuity of the constructed 3-D volume (Figure 7) is amplified by using their MAP estimates [51]:

$$
\begin{aligned}
\hat{\delta}_{x, y, z}= & \arg \min _{\tilde{\delta}_{x, y, z}}\left\{\left|\delta_{x, y, z}-\tilde{\delta}_{x, y, z}\right|^{\alpha}\right. \\
& \left.+\rho^{\alpha} \lambda^{\beta} \sum_{\left(x^{\prime}, y^{\prime}, z^{\prime}\right) \in V_{(x, y, z)}} \eta_{(x, y, z),\left(x^{\prime}, y^{\prime}, z^{\prime}\right)}\left|\tilde{\delta}_{x, y, z}-\delta_{x^{\prime}, y^{\prime}, z^{\prime}}\right|^{\beta}\right\}
\end{aligned}
$$

where $\delta_{x, y, z}$ and $\tilde{\delta}_{x, y, z}$ denote the original values and their expected estimates, $v_{(x, y, z)}$ is the 26-neighborhood voxel set (Figure 7), $\eta_{(x, y, z),\left(x^{\prime}, y^{\prime}, z^{\prime}\right)}$ is the GGMRF potential, and $\rho$ and $\lambda$ are scaling factors. The parameter $\beta \in[1.01,2.0]$ controls the level of smoothing (e.g., smooth, $\beta=2$, vs. relatively abrupt edges, $\beta=1.01$ ). The parameter $\alpha \in\{1,2\}$ determines the Gaussian, $\alpha=2$, or Laplace, $\alpha=2$, prior distribution of the estimator. Then, the color maps are generated based on the final estimated $\tilde{\delta}$ (see Figure 8).

Finally, to allocate the boundary of the detected tumors, which is important to determine the cancer stage in case of malignancy, we used a level set-based deformable model controlled by a stochastic speed function [59]. The latter accounts for the perfusion information and

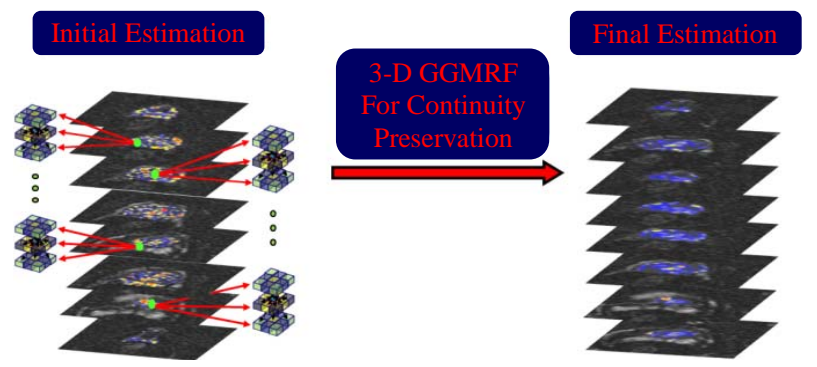

Figure 7. Enhanced perfusion estimation and continuity analysis using the 3-D GGMRF image model.

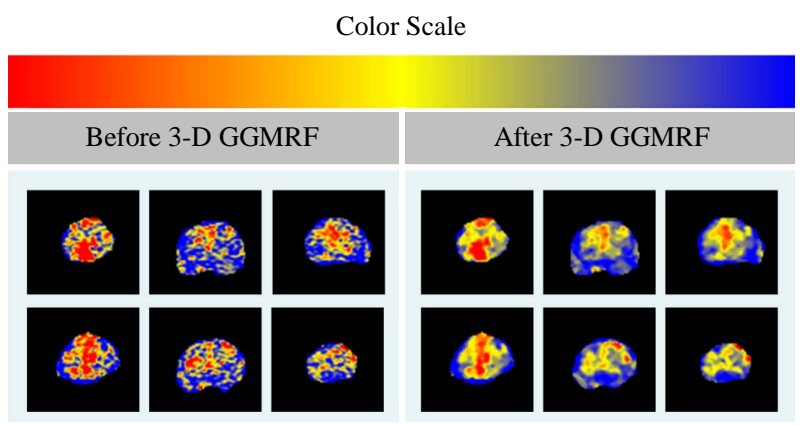

Figure 8. Color-coded maps for three of the test subjects (columnwise) before and after the 3-D GGMRF smoothing with $\rho$ $=1, \lambda=5, \beta=1: 01, \alpha=2$, and $\eta_{(x, y, z),\left(x^{\prime}, y^{\prime}, z^{\prime}\right)}=\sqrt{2}$ and their respective color-coded maps. The red and blue ends of the color scale relate to the maximum and minimum changes, respectively.

spatial interactions between the prostate voxels.

\section{EXPERIMENTAL RESULTS}

The performance of the proposed framework has been evaluated by applying it on DWI prostate images collected from 28 patients. These patients had biopsyproven prostate cancer and underwent DWI at 1.5-T (SIGNA Horizon, General Electric Medical Systems, Milwaukee, WIS). DWI were then obtained using monodirectional gradients and a multi-section Fast Spin Echo type (FSE) echo-planar sequence in the axial plane using a body coil with the following imaging parameters: TE: 84:6 ms; TR: 8.000 ms; Band Width $142 \mathrm{kHz}$; FOV 34 $\mathrm{cm}$; slice thickness $3 \mathrm{~mm}$; inter-slice gap $0 \mathrm{~mm}$; seven excitations, water excitation with $b$ value of $0 \mathrm{~s} / \mathrm{mm}^{2}$ and $800 \mathrm{~s} / \mathrm{mm}^{2}$. Fifty four slices were obtained in 120 second. to cover the prostate in each patient. Note all the subjects were diagnosed using a biopsy (ground truth).

\subsection{Segmentation Results}

The proposed segmentation approach has been tested on 28 independent data sets of DWI images. Figure 9 shows sample examples of prostate segmentation from different data sets, with respect to the ground truth segmentation. The ground truths were obtained by manual delineation of the prostate borders by an MR imaging expert. To highlight the advantages of our segmentation technique, we compare it to the shape-based segmentation approach proposed by Tsai et al. [60]. We re-implemented the method described in [60] and tested it on our locallyacquired data.

Figure 9 compares qualitatively the accuracy of our approach and the shape-based approach [60] with respect to the ground truth. The segmentation accuracy for all data sets has been evaluated using the average segmentation error, given by Eq.7. Differences between the mean 

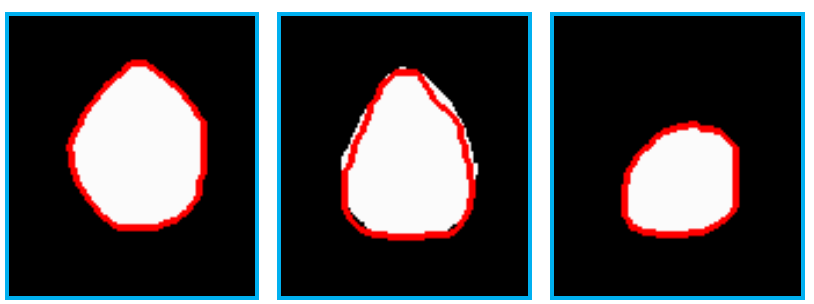

(a)
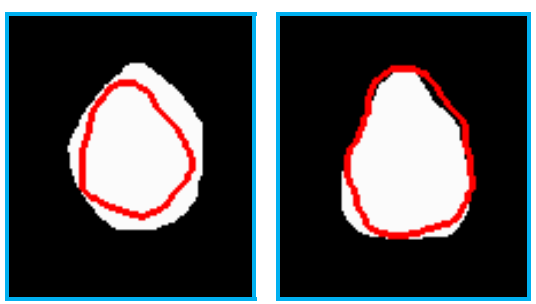

(b)
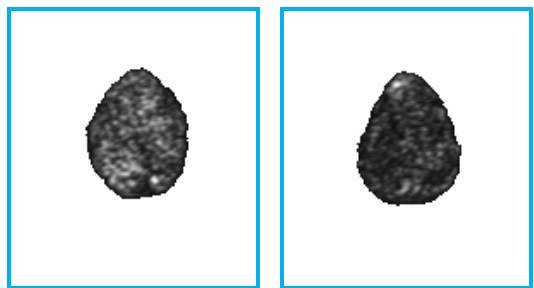

(c)

Figure 9. 3-D prostate segmentation projected onto 2-D. (a) Our segmentation (red) in comparison with the ground truth (white); (b) The segmentation with the algorithm in [60] (red) comparison with the ground truth; and (c) 2-D visualization for our segmented prostates for three of the test subjects.

errors for our segmentation and the shape-based approach [60] in Table 1 are statistically significant by the unpaired t-test and thus highlight the advantages of the proposed integration of the shape prior, prostate/background marginal intensity distributions, and spatial interaction characteristics into MAP-based segmentation.

Moreover, the accuracy of our segmentation approach has been evaluated, with respect to the expert tracing, using the PPV, Sens, DSC [61], and the APD between the borders of ground truth $G$ and automatic segmentation $C$ (see Figure 10). Table 2 compares the segmentation over all the test data sets with the ground truth obtained by manual tracing by an imaging expert.

\subsection{Diagnostic Results}

The ultimate goal of the proposed framework is to distinguish between benign and malignant detected tumors. The malignant tissues show higher signal intensity with a $b$-value of $800 \mathrm{~s} / \mathrm{mm}^{2}$, and a lower Apparent Diffusion Coefficient (ADC) compared with benign and normal tissue due to the replacement of normal tissue. To distinguish between the benign and malignant cases, we used a KNN classifier learning statistical characteristics
Table 1. A comparative segmentation accuracy over all test data sets for our approach and [60]. Note that "STD" stands for standard deviation.

\begin{tabular}{ccc}
\hline & $E_{\text {avg }} \%$ & \\
& \multicolumn{3}{c}{ Algorithm } \\
\cline { 2 - 3 } & Our & {$[60]$} \\
\hline Min. Error \% & 0 & 0 \\
Max. Error \% & 1.6005 & 2.7724 \\
Mean Error \% & 0.5500 & 1.4615 \\
STD. \% & 0.3085 & 0.7687 \\
P-value & & 0.0001 \\
\hline
\end{tabular}

Table 2. Error statistics over all test data sets. Note that "STD" stands for standard deviation and "APD" values are in mm.

\begin{tabular}{ccccc}
\hline & \multicolumn{4}{c}{ Performance measures } \\
\cline { 2 - 5 } & PPV & Sens & DSC & APD \\
\hline Min. & 0.857 & 0.882 & 0.841 & 0.00 \\
Max & 0.991 & 0.851 & 0.930 & 3.1 \\
Mean & 0.952 & 0.816 & 0.991 & 0.60 \\
STD. & 0.004 & 0.004 & 0.004 & 0.80 \\
\hline
\end{tabular}

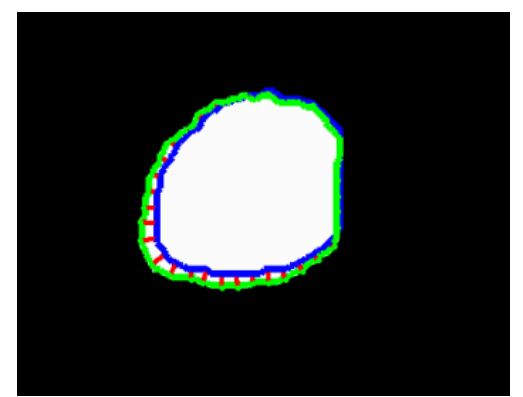

Figure 10. Prostate image with ground truth (blue) and automatic segmentation (green) contours, and their associated streamlines (red) obtained by the solution of the Laplace equation yielding the estimation of the APD.

of the DWI. The characteristics are obtained from the training sets containing both benign and malignant cases. After training, three features namely are the mean intensity value of the DWI at $0 \mathrm{~s} / \mathrm{mm}^{2}$, the mean intensity value of the DWI at $800 \mathrm{~s} / \mathrm{mm}^{2}$, and the mean value of ADC maps [62], were chosen to classify the test cases.

To build the KNN classifier that characterizes the prostate tissue, we used 13 subjects for training, and the other 15 subjects for testing. The diagnostic accuracy based on the combined three features resulted in correct classifications of all 28 data sets (i.e., $100 \%$ accuracy). 

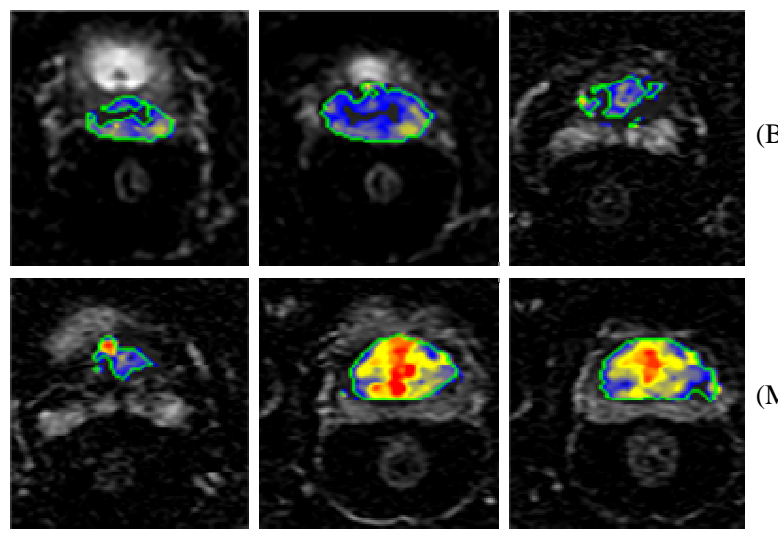

(B)

Figure 11. Tumor's contour determination (green) using the level set approach for multiple image sections for benign (B) and malignant $(\mathrm{M})$ subjects.

For regional display we explore pixel-by-pixel maps of the registered diffusion data. The diffusion was computed for each pixel and superimposed on an image slice to form a parametric image. Also, for visual assessment of the prostate tumor the tumor contours are determined. Figure 11 shows the tumor contours determination for selected image sections for two subjects involved in our study.

\section{CONCLUSIONS AND DISCUSSION}

In this paper, we present a novel fully automatic framework for detecting prostate cancer using DWI. The framework includes prostate segmentation, nonrigid registration, and KNN-based classification. For prostate segmentation, we introduced a new $3 \mathrm{D}$ approach that is based on a MAP estimate of a new log-likelihood function that accounts for the shape priori, the spatial interaction, and the current appearance of the prostate and its background which increases the accuracy of automatic segmentation, evidenced by the error and the DSC analysis (Tables 1 and 2). Following segmentation, we used a nonrigid registration approach that deforms the prostate object on iso-contours instead of a square lattice, which provides higher degrees of freedom to obtain accurate deformation. In the classification step, the segmented prostate regions are classified into malignant or benign using the KNN classifier. Applications of the proposed framework can assist the radiologist in detecting all prostate cancer locations and could replace the use of current technologies to determine the type of prostate cancer.

Although we have obtained promising results in this initial study using DWI data in 28 patients, potential widespread adoption would require confirmation by other groups, and investigation in a larger number of subjects. Our future work will focus on comparing the diagnostic accuracy of prostate cancer detection using other imaging modalities, such as DCE-MRI.

\section{REFERENCES}

[1] Siegel, R., DeSantis, C., Virgo, K., Stein, K., Mariotto, A., Smith, T., et al. (2012) Cancer treatment and survivorship statistics. A Cancer Journal for Clinicians, 62, 220-241.

[2] American Cancer Society (2012) Cancer treatment and survivorship facts \& figures 2012-2013. http://www.cancer.org/acs

[3] Deem, S., DeFade, B., Lohri, J., Tierney, J., Modak, A. and Emmett, M. (2010) Prostate cancer screening: A primary care survey. Health, 2, 1179-1183. doi:10.4236/health.2010.210173

[4] Stenman, U.H., Leinonen, J., Zhang, W.M. and Finne, P. (1999) Prostate-specific antigen. Seminars in Cancer Bio$\log y$, 9, 83-93. doi:10.1006/scbi.1998.0086

[5] Halpern, E.J. (2006) Contrast-enhanced ultrasound imaging of prostate cancer. Urology, 8, 29-37.

[6] Ives, E.P., Burke, M.A., Edmonds, P.R., Gomella, L.G. and Halpern, E.J. (2005) Quantitative computed tomography perfusion of prostate cancer: Correlation with whole-mount pathology. Clinical Prostate Cancer, 4, 109112. doi:10.3816/CGC.2005.n.018

[7] Shah, N., Sattar, A., Benanti, M., Hollander, S. and Cheuck, L. (2006) Magnetic resonance spectroscopy as an imaging tool for cancer: A review of the literature. Journal of the American Osteopathic Association, 106, 23-27.

[8] Noworolski, S.M., Henry, R.G., Vigneron, D.B. and Kurhanewicz, J. (2005) Dynamic contrast-enhanced MRI in normal and abnormal prostate tissues as defined by biopsy, MRI, and 3D MRSI. Magnetic Resonance in Medicine, 53, 249-255. doi:10.1002/mrm.20374

[9] Kajihara, H., Hayashida, Y., Murakami, R., Katahira, K., Nishimura, R., Hamada, Y., et al. (2009) Usefulness of diffusion-weighted imaging in the localization of prostate cancer. International Journal of Radiation Oncology Biology Physics, 74, 399-403.

doi:10.1016/j.ijrobp.2008.08.017

[10] Ellis, W.J. and Brawer, M.K. (1995) Repeat prostate needle biopsy: Who needs it? Journal of Urology, 153, 1496-1498. doi:10.1016/S0022-5347(01)67444-5

[11] Applewhite, J.C., Matlaga, B.R., McCullough, D.L. and Hall, M.C. (2001) Transrectal ultrasound and biopsy in the early diagnosis of prostate cancer. Cancer Control Journal, 8, 141-150.

[12] Gossner, J. (2012) Computed tomography of the prostateA review. The Internet Journal of Radiology, 14, 1.

[13] Bouchelouche, K., Turkbey, B., Choyke, P. and Capala, J. (2010) Imaging prostate cancer: An update on positron emission tomography and magnetic resonance imaging. Current Urology Reports, 11, 180-190. doi:10.1007/s11934-010-0105-9

[14] Fuchsjager, M., Shukla-Dave, A., Akin, O., Barentsz, J. and Hricak, H. (2008) Prostate cancer imaging. Acta Radiologica, 49, 107-120. doi:10.1080/02841850701545821

[15] Choi, Y.J., Kim, J.K., Kim, N., Kim, K.W., Choi, E.K. 
and Cho, K.S. (2007) Functional MR imaging of prostate cancer. RadioGraphics, 27, 63-75.

doi:10.1148/rg.271065078

[16] Zakian, K.L., Eberhardt, S., Hricak, H., Shukla-Dave, A., Kleinman, S., Muruganandham, M., et al. (2003) Transition zone prostate cancer: Metabolic characteristics at $1 \mathrm{H}$ MR spectroscopic imaging-initial results. Radiology, 229, 241-247. doi:10.1148/radiol.2291021383

[17] Punwani, S., Emberton, M., Walkden, M., Sohaib, A., Freeman, A., Ahmed, H., et al. (2012) Prostatic cancer surveillance following whole-gland high-intensity focused ultrasound: Comparison of MRI and prostate-specific antigen for detection of residual or recurrent disease. British Journal of Radiology, 85, 720-728. doi:10.1259/bjr/61380797

[18] Bhave, G., Lewis, Julia, B. and Chang, S. (2008) Association of gadolinium based magnetic resonance imaging contrast agents and nephrogenic systemic fibrosis. Journal of Urology, 180, 830-835. doi:10.1016/j.juro.2008.05.005

[19] Yu, K.K. and Hricak, H. (2000) Imaging prostate cancer. Radiologic Clinics of North America, 38, 59-85. doi:10.1016/S0033-8389(05)70150-0

[20] Ikonen, S., Karkkainen, P., Kivisaari, L., Salo, J.O., Taari, K., Vehmas, T., et al. (2001) Endorectal magnetic re-sonance imaging of prostatic cancer: Comparison between fat-suppressed T2-weighted fast spin echo and three-dimensional dual-echo, steady-state sequences. European Radiology, 11, 236-241. doi:10.1007/s003300000598

[21] Shimofusa, R., Fujimoto, H., Akamata, H., Motoori, K., Yamamoto, S., Ueda, T., et al. (2005) Diffusion-weighted imaging of prostate cancer. Journal of Computer Assisted Tomography, 29, 149-153. doi:10.1097/01.rct.0000156396.13522.f2

[22] Hacklander, T., Scharwachter, C., Golz, R. and Mertens, H. (2006) Value of diffusion-weighted imaging for diagnosing vertebral metastases due to prostate cancer in comparison to other primary tumors. Rofo, 178, 416-424.

[23] Yoshimitsu, K., Kiyoshima, K., Irie, H., Tajima, T., Asayama, Y., Hirakawa, M., et al. (2008) Usefulness of apparent diffusion coefficient map in diagnosing prostate carcinoma: Correlation with stepwise histopathology. Journal of Magnetic Resonance Imaging, 27, 132-139. doi:10.1002/jmri.21181

[24] Lim, H.K., Kim, J.K., Kim, K.A. and Cho, K.S. (2009) Prostate cancer: Apparent diffusion coefficient map with T2-weighted images for detection a multireader study. Radiology, 250, 145-151. doi:10.1148/radiol.2501080207

[25] Haider, M.A., van der Kwast, T.H., Tanguay, J., Evans, A.J., Hashmi, A.T., Lockwood, G., et al. (2007) Combined T2-weighted and diffusion-weighted MRI for localization of prostate cancer. American Journal of Roentgenology, 189, 323-328. doi:10.2214/AJR.07.2211

[26] Padhani, A.R., Liu, G., Koh, D.M., Chenevert, T.L., Thoeny, H.C., Takahara, T., et al. (2009) Diffusionweighted magnetic resonance imaging as a cancer biomarker: Consensus and recommendations. Neoplasia, 11, 102-125.

[27] Kitajima, K., Kaji, Y., Fukabori, Y., Yoshida, K., Suga- numa, N. and Sugimura, K. (2010) Prostate cancer detection with 3T MRI: Comparison of diffusion-weighted imaging and dynamic contrast-enhanced MRI in combination with T2-weighted imaging. Journal of Magnetic Resonance Imaging, 31, 625-631. doi:10.1002/jmri.22075

[28] Iwazawa, J., Mitani, T., Sassa, S. and Ohue, S. (2011) Prostate cancer detection with MRI: Is dynamic contrastenhanced imaging necessary in addition to diffusionweighted imaging? Diagnostic and Interventional Radiology, 17, 243-248.

[29] Kim, C.K., Park, B.K. and Lee, H.M. (2009) Prediction of locally recurrent prostate cancer after radiation therapy: Incremental value of 3T diffusion-weighted MRI. Journal of Magnetic Resonance Imaging, 29, 391-397. doi:10.1002/jmri.21645

[30] Tan, C.H., Wang, J. and Kundra, V. (2011) Diffusion weighted imaging in prostate cancer. European Radiology, 21, 593-603. doi:10.1007/s00330-010-1960-y

[31] Chan, I., Wells, III, W., Mulkern, R.V., Haker, S., Zhang, J., Zou, K.H., et al. (2003) Detection of prostate cancer by integration of line-scan diffusion, T2-mapping and T2-weighted magnetic resonance imaging; a multichannel statistical classifier. Journal of Medical Physics, 30, 23902398. doi:10.1118/1.1593633

[32] Huisman, H., Vos, P., Litjens, G., Hambrock, T. and Barentsz, J. (2010) Computer aided detection of prostate cancer using t2w, DWI and DCE-MRI: Methods and clinical applications. Proceedings of International Workshop, Held in Conjunction with MICCAI 2010, Beijing, 20-24 September 2010, 4-14.

[33] Frangi, A.F., Niessen, W.J., Vincken, K.L. and Viergever, M.A. (1998) Multiscale vessel enhancement filtering. Proceedings of the 1st Annual International Conference of the MICCA, Cambridge, 11-13 October 1998, 130-137.

[34] Viswanath, S., Bloch, B.N., Genega, E., Rofsky, N., Lenkinski, R., Chappelow, J., et al. (2008) A comprehensive segmentation, registration, and cancer detection scheme on 3 Tesla in vivo prostate DCE-MRI. Medical Image Computing and Computer-Assisted Intervention, 11, 662 669.

[35] Langer, Deanna, L., van der Kwast Theodorus, H., Evans, Andrew, J., et al. (2009) Prostate cancer detection with multi-parametric MRI: Logistic regression analysis of quantitative T2, diffusion-weighted imaging, and dynamic contrast-enhanced MRI. Journal of Magnetic Resonance Imaging, 30, 327-334. doi:10.1002/jmri.21824

[36] Villeirs, G.M., L Verstraete, K., De Neve, W.J. and De Meerleer, G.O. (2005) Magnetic resonance imaging anatomy of the prostate and periprostatic area: A guide for radiotherapists. Radiotherapy and Oncology, 76, 99-106. doi:10.1016/j.radonc.2005.06.015

[37] Zhan, Y. and Shen, D. (2003) Automated segmentation of 3D US prostate images using statistical texture-based matching method. Proceedings of the 6th Annual International Conference of the MICCA, Montreal, 15-18 November 2003, 2878, 688-696.

[38] Lixin, G., Pathak, S.D., Haynor, D.R., Cho, P.S. and Kim, Y. (2004) Parametric shape modeling using deformable superellipses for prostate segmentation. IEEE Transac- 
tions on Medical Imaging, 23, 340-349. doi:10.1109/TMI.2004.824237

[39] Zwiggelaar, R., Zhu, Y. and Williams, S. (2003) Semiautomatic segmentation of the prostate. Pattern Recognition and Image Analysis, 2652, 1108-1116. doi:10.1007/978-3-540-44871-6_128

[40] Zhu, Y., Williams, S. and Zwiggelaar, R. (2004) Segmentation of volumetric prostate MRI data using hybrid 2d+3d shape modeling. Medical Image Understanding and Analysis, 61-64.

[41] Toth, R., Tiwari, P., Rosen, M., Kalyanpur, A., Pungavkar, S. and Madabhushi, A. (2008) A multi-modal prostate segmentation scheme by combining spectral clustering and active shape models. Proceedings of the International Society of Optics and Photonic, San Jose, 69144S-69144S.

[42] Klein, S., van der Heide, U.A., Raaymakers, B.W., Kotte, A.N.T.J., Staring, M. and Pluim, J.P.W. (2007) Segmentation of the prostate in MR images by atlas matching. Proceedings of the 4th Annual IEEE International Symposium on Biomedical Imaging: From Nano to Macro (ISBI), Washington DC, 12-15 April 2007, 1300-1303.

[43] Vikal, S., Haker, S., Tempany, C. and Fichtinger, G. (2009) Prostate contouring in MRI guided biopsy. Proceedings of the International Society of Optics and Photonics, 7259, 72594A.

[44] Martin, S., Troccaz, J. and Daanenc, V. (2010) Automated segmentation of the prostate in 3D MR images using a probabilistic atlas and a spatially constrained deformable model. Journal of Medical Physics, 37, 15791590. doi:10.1118/1.3315367

[45] Hambrock, T., Somford, D.M., Hoeks, C., Bouwense, S. A., Huisman, H., Yakar, D., et al. (2010) Magnetic resonance imaging guided prostate biopsy in men with repeat negative biopsies and increased prostate specific antigen. Journal of Urology, 183, 520-527.

doi:10.1016/j.juro.2009.10.022

[46] Firjani, A., Khalifa, F., Elnakib, A., Gimel’farb, G., ElGhar, M.A., Elmaghraby, A. and El-Baz, A. (2011) 3D automatic approach for precise segmentation of the prostate from diffusion-weighted magnetic resonance imaging. Proceedings of 18th Annual IEEE International Conference on Image Processing (ICIP), Brussels, 11-14 September 2011, 2285-2288.

[47] Firjani, A., Khalifa, F., Elnakib, A., Gimel’farb, G., ElGhar, M.A., Elmaghraby, A. and El-Baz, A. (2012) A novel image-based approach for early detection of prostate cancer. Proceedings of 19th Annual IEEE International Conference on Image Processing (ICIP), Orlando, 30 September-3 October 2012, 2849-2852.

[48] Firjani, A., Elnakib, A., Khalifa, F., Gimel’farb, G., ElGhar, M.A., Elmaghraby, A. and El-Baz, A. (2011) A new 3D automatic segmentation framework for accurate extraction of prostate from diffusion imaging. Proceedings of 3rd Annual Biomedical Sciences and Engineering Conference (BSEC), Knoxville, 15-17 March 2011, 1-4.

[49] El-Baz, A., Gimel'farb, G., Falk, R., Holland, T. and Shaffer, T. (2008) A new stochastic framework for accurate lung segmentation. Proceedings of the 11th Annual International Conference of the MICCA, New York, 6-10 Sep- tember 2008, 322-330.

[50] El-Baz, A., Elnakib, A., Khalifa, F., Abou El-Ghar, M., McClure, P., Soliman, A. and Gimel'farb, G. (2012) Precise segmentation of 3-D magnetic resonance angiography. IEEE Transactions on Biomedical Engineering, 7, 2019-2029. doi:10.1109/TBME.2012.2196434

[51] El-Baz, A. and Gimel'farb, G. (2007) EM based approximation of empirical distributions with linear combinations of discrete Gaussians. Proceedings of 14th Annual IEEE International Conference on Image Processing (ICIP), San Antonio, 16-19 September 2007, 4, 373-376.

[52] Farag, A.A., El-Baz, A. and Gimel'farb, G. (2006) Precise segmentation of multi-modal images. IEEE Transactions on Image Processing, 15, 952-968. doi:10.1109/TIP.2005.863949

[53] El-Baz, A. (2006) Novel stochastic models for medical image analysis. Ph.D. Thesis, University of Louisville, Louisville.

[54] Viola, P. and Wells, W.M. (1997) Alignment by maximization of mutual information. International Journal of Computer Vision, 24, 137-154. doi:10.1023/A:1007958904918

[55] Besag, J. (1986) On the statistical-analysis of dirty pictures. Journal of the Royal Statistical Society Series BMethodological, 48, 259-302.

[56] Khalifa, F., El-Baz, A., Gimel'farb, G. and Abu El-Ghar. M. (2010) Non-invasive image-based approach for early detection of acute renal rejection. Proceedings of the 13th Annual International Conference of the MICCA, Beijing, 20-24 September 2010, 1, 10-18.

[57] Patterson, D.M., Padhani, A.R. and Collins, D.J. (2008) Technology insight: Water diffusion MRI-A potential new biomarker of response to cancer therapy. Nature Clinical Practice Oncology, 5, 220-233. doi:10.1038/ncponc1073

[58] Bouman, C. and Sauer, K. (1993) A generalized Gaussian image model for edge-preserving MAP estimation. IEEE Transactions on Image Processing, 2, 296-310. doi:10.1109/83.236536

[59] Khalifa, F., Beache, G.M., Gimelrfarb, G., Giridharan, G.A. and El-Baz, A. (2012) Accurate automatic analysis of cardiac cine images. IEEE Transactions on Biomedical Engineering, 59, 445-455. doi:10.1109/TBME.2011.2174235

[60] Tsai, A., Yezzi, Jr., A., Wells, W., Tempany, C., Tucker, D., Fan, A., et al. (2003) A shape-based approach to the segmentation of medical imagery using level sets. IEEE Transactions on Medical Imaging, 22, 137-154. doi:10.1109/TMI.2002.808355

[61] Dice, L.R. (1945) Measures of the amount of ecologic association between species. Ecology, 26, 297-302. doi:10.2307/1932409

[62] Walker-Samuel, S., Orton, M., Boult, J.K. and Robinson, S.P. (2011) Improving apparent diffusion coefficient estimates and elucidating tumor heterogeneity using Bayesian adaptive smoothing. Magnetic Resonance in Medicine, 65, 438-447. doi:10.1002/mrm.22572 